\title{
Characterisation of ultra- and nanofiltration membranes for predictive purposes - development of a model for hindered transport of uncharged solutes in asymmetric pores
}

\author{
A. Morão* ${ }^{a *}$ M.T. Pessoa de Amorim ${ }^{\mathrm{b}}$, A. Lopes ${ }^{\mathrm{a}}$, I.C. Gonçalves ${ }^{\mathrm{a}}$ \\ ${ }^{a}$ Department of Chemistry, University of Beira Interior 6201-001 Covilhã, Portugal \\ email:ammorao@ubi.pt \\ ${ }^{b}$ Department of Textile Engineering, University of Minho - 4800-058 Guimarães , Portugal
}

Received 22 October 2005; accepted 4 March 2006

\section{Introduction}

Several works have reported the characterisation of membranes with uncharged solutes using models of hindered transport to describe the mass transfer processes inside ultra- and nanofiltration membranes [1-6]. These models are usually applied to predict intrinsic rejections of solute of known radius, after characterisation of the membranes with reference solutes.

It is generally assumed that the membranes consist of a porous matrix of cylindrical (thus symmetric) pores. However, it is well known that, in general, commercially available ultraand nanofiltration membranes have an asymmetric structure. Even in those cases where there is a thin skin (porous or homogeneous) of different material on the top of the porous support (composite membranes), it is not clear whether the top region of the support should be included or not in the so-called "active layer" of the membrane. In both cases an asymmetric structure may be present. Thus, it is important to

*Corresponding author. understand and quantify the effect of the asymmetry on the rejections.

It was the aim of this work to develop a model as much as simple that quantifies this effect, and apply this model to a practical problem of the characterisation of a commercial nanofiltration membrane.

\section{Theory: development of the model}

The introduction of asymmetry in the pores, by changing the cylinders to conical surfaces poses serious theoretical problems, since the basic available equations describing the transport of the solute inside the pore, assume constant velocity of the species (solute and solvent), and a balance of forces acting on the solute [5]. However, in a conical pore the velocities are decreasing along the pore, and thus, the forces are not balanced. Apart from this, the hydrodynamic models generally used to quantify the effects of the pore wall on the solute transport [2,4] are only applicable to long (infinite) cylindrical pores.

To avoid these difficulties, the asymmetric pore is assumed to consist of a various (four) 


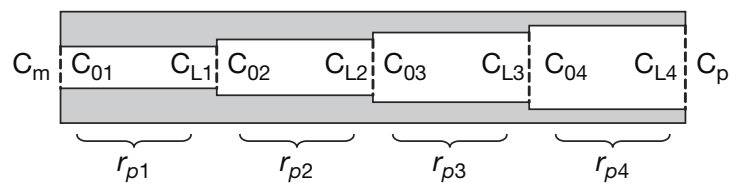

Fig. 1. Model of the "asymmetric" pore. Concentrations at the various interfaces, where partition occurs are specified, and the radius of the various cylinders.

consecutive cylinders (Fig. 1). The number of cylinders should not be too large to ensure the applicability of the approximation of infinite pores. The transport equations used in each cylindrical segment are similar to those proposed by Bowen and Welfoot [1]. The major differences are in the expressions to calculate the partition coefficients of the solute along the pore, and the expressions to calculate the concentrations indicated at the interfaces and permeate (see Fig. 1). The partition coefficients are given by

$$
\begin{aligned}
& \Phi_{1}=\frac{C_{01}}{C_{m}}=\left(1-\lambda_{1}\right)^{2} \quad \Phi_{2}=\frac{C_{02}}{C_{L 1}}=\frac{\left(1-\lambda_{2}\right)^{2}}{\left(1-\lambda_{1}\right)^{2}} \\
& \Phi_{3}=\frac{C_{03}}{C_{L 2}}=\frac{\left(1-\lambda_{3}\right)^{2}}{\left(1-\lambda_{2}\right)^{2}} \quad \Phi_{4}=\frac{C_{04}}{C_{L 3}}=\frac{\left(1-\lambda_{4}\right)^{2}}{\left(1-\lambda_{3}\right)^{2}} \\
& \Phi_{5}=\frac{C_{p}}{C_{L 4}}=\frac{1}{\left(1-\lambda_{4}\right)^{2}}
\end{aligned}
$$

and the concentrations by

$$
\begin{aligned}
& \langle C\rangle_{L i}=\frac{\left[\left(K_{c i}-Y_{i}\right)\langle C\rangle_{0 i}-C_{p}\right] \exp \left(P e_{i}^{\prime}\right)+C_{p}}{K_{c i}-Y_{i}} \\
& P e_{i}^{\prime}=\frac{\left(K_{c i}-Y_{i}\right) r_{p i}^{2} \Delta P_{e i}}{8 \eta_{0} K_{d i} D_{\infty}} \quad i=1,2,3,4
\end{aligned}
$$

The asymmetry of the pore is defined by a parameter, $H$, that defines the radius of the cylinders in each segment:

$r_{p i}=\left[\left(\frac{i-1}{3}\right) H+1\right] r_{p} \quad$ with $i=1,2,3$ and 4
The segments may be assumed to have the same length, or the length of each segment calculated assuming a particular set of $\Delta P_{e i}$ values. The total length of the "active layer" (pore length) is not a parameter of the model, and may be evaluated from the $r_{p}, H, \Delta P_{e}$, the hydraulic permeability of the membrane and its surface porosity.

\section{Simulations}

It is presented as a series of simulations of the effect of the asymmetry on the rejections of solutes of different size, in membranes with the same pore radius on the top layer, and the same pore length. The results show that rejections are decreased by the introduction of asymmetry, especially for low fluxes and when the ratio $r_{s} / r_{p}$ is low.

\section{Characterisation of a nanofiltration membrane}

A commercial nanofiltration membrane (NFT-50 from DSS/Alfa Laval) was characterised in terms of pore radius at the surface $\left(r_{p}\right)$ and asymmetry $(H)$ using two different uncharged solutes (glycerol and ethylene glycol), by application of the model of asymmetric pores. Assuming the same pressure drop for each segment, the results show an excellent agreement in terms of the fitted parameters $\left(r_{p}\right.$ and $H$ ) when comparing the results obtained with the two reference solutes.

\section{Acknowledgements}

Fundação para a Ciência e a Tecnologia (FCT) - The grant awarded to António Morão is gratefully acknowledged (SFRH/BD/6485/2001).

\section{References}

[1] W.R. Bowen and J.S. Welfoot, Chem. Eng. Sci., 57 (2002) 1121-1137. 
[2] W.R. Bowen and A.O. Sharif, J. Colloid Interf. Sci., 168 (1994) 414-421.

[3] C. Combe, C. Guizard, P. Aimar and V. Sanchez, J. Membr. Sci., 129(2) (1997), 147.

[4] W.M. Deen, AIChE J., 9 (1987) 1409-1425.
[5] T.R. Noordman and J.A. Wesselingh, J. Membr. Sci., 210 (2002) 227-243.

[6] M.J. Rosa, PhD Thesis, Instituto Superior Técnico, 1995. 